\title{
Multiparameter Higher Order Daehee and Bernoulli Numbers and Polynomials
}

\author{
Beih S. El-Desouky, Abdelfattah Mustafa, Fatma M. Abdel-Moneim \\ Department of Mathematics, Faculty of Science, Mansoura University, Mansoura, Egypt \\ Email: b_eldesouky@yahoo.com, abdelfatah_mustafa@yahoo.com, amelsayed@mans.edu.eg, queen_evry@yahoo.com
}

How to cite this paper: El-Desouky, B.S., Mustafa, A. and Abdel-Moneim, F.M. (2017) Multiparameter Higher Order Daehee and Bernoulli Numbers and Polynomials. Applied Mathematics, 8, 775-785. https://doi.org/10.4236/am.2017.86060

Received: March 17, 2017

Accepted: June 5, 2017

Published: June 8, 2017

Copyright (C) 2017 by authors and Scientific Research Publishing Inc. This work is licensed under the Creative Commons Attribution International License (CC BY 4.0).

http://creativecommons.org/licenses/by/4.0/

\begin{abstract}
This paper gives a new generalization of higher order Daehee and Bernoulli numbers and polynomials. We define the multiparameter higher order Daehee numbers and polynomials of the first and second kind. Moreover, we derive some new results for these numbers and polynomials. The relations between these numbers and Stirling and Bernoulli numbers are obtained. Furthermore, some interesting special cases of the generalized higher order Daehee and Bernoulli numbers and polynomials are deduced.
\end{abstract}

\section{Keywords}

Daehee Numbers, Daehee Polynomials, Higher-Order Daehee Numbers, Higher-Order Daehee Polynomials, Higher-Order Bernoulli Polynomials, Multiparities Daehee Polynomials

\section{Fundamental and Principles}

The $n$-th Daehee polynomials are defined by [1]-[9].

$$
\left(\frac{\log (1+t)}{t}\right)(1+t)^{x}=\sum_{n=0}^{\infty} D_{n}(x) \frac{t^{n}}{n !} .
$$

If $x=0$ hence $D_{n}=D_{n}(0)$ are called Daehee numbers,

For $n \geq 0$,

$$
\int_{\mathbb{Z}_{p}}(x)_{n} \mathrm{~d} \mu_{0}(x)=D_{n} .
$$

For $k \in \mathbb{N}$, Kim [1] introduced Daehee numbers of the first kind of order $k$ by

$$
D_{n}^{(k)}=\int_{\mathbb{Z}_{p}} \int_{\mathbb{Z}_{p}} \cdots \int_{\mathbb{Z}_{p}}\left(x_{1}+x_{2}+\cdots+x_{k}\right)_{n} \mathrm{~d} \mu\left(x_{1}\right) \mathrm{d} \mu\left(x_{2}\right) \cdots \mathrm{d} \mu\left(x_{k}\right),
$$

where $n$ is nonnegative integer. 
The generating function of these numbers are given by

$$
\sum_{n=0}^{\infty} D_{n}^{(k)} \frac{t^{n}}{n !}=\left(\frac{\log (1+t)}{t}\right)^{k},
$$

where $n \in \mathbb{Z} \geq 0, k \in \mathbb{N}$.

The higher-order Daehee polynomials are defined by, [10]

$$
D_{n}^{(k)}(x)=\int_{\mathbb{Z}_{p}} \int_{\mathbb{Z}_{p}} \cdots \int_{\mathbb{Z}_{p}}\left(x_{1}+x_{2}+\cdots+x_{k}+x\right)_{n} \mathrm{~d} \mu\left(x_{1}\right) \mathrm{d} \mu\left(x_{2}\right) \cdots \mathrm{d} \mu\left(x_{k}\right) .
$$

For $k \in \mathbb{Z}$, the Bernoulli polynomials of order $k$ are defined by, see [1] [11] [12] [13],

$$
\left(\frac{t}{\left(\mathrm{e}^{t}-1\right)^{k}}\right) \mathrm{e}^{\mathrm{xt}}=\sum_{n=0}^{\infty} B_{n}^{(k)}(x) \frac{t^{n}}{n !},
$$

when $x=0, B_{n}^{(k)}=B_{n}^{(k)}(0)$ are called the Bernoulli numbers of order $k$.

Also, Kim proved that

$$
D_{n}^{(k)}(x)=\sum_{\ell=0}^{n} s(n, \ell) B_{\ell}^{(k)}(x)
$$

and

$$
B_{n}^{(k)}(x)=\sum_{\ell=0}^{n} S(n, \ell) D_{\ell}^{(k)}(x) .
$$

An explicit formula for higher-order Daehee numbers are given by

$$
D_{n}^{(k)}=\frac{s(n+k, k)}{\left(\begin{array}{c}
n+k \\
k
\end{array}\right)}, \quad(n \geq 0, k \geq 1)
$$

where $s(n, k)$ are the Stirling numbers of the first kind, see [1] [10].

In this article, Sections 2 and 3, give a new generalization of higher order Daehee numbers and polynomials which are called the multiparameter higher order Daehee numbers and polynomials of the first kind. In Sections 4 and 5, we define the multiparameter higher order Daehee numbers and polynomials of the second kind. Furthermore, the relations between these numbers and Stirling and Bernoulli numbers are obtained.

\section{Multiparameter Higher Order Daehee Numbers of the First Kind}

The multiparameter higher order Daehee numbers of the first kind $D_{n ; \bar{\alpha}, \bar{r}}^{(k)}$ are defined by

$$
D_{n ; \bar{\alpha}, \bar{r}}^{(k)}=\int_{\mathbb{Z}_{p}} \int_{\mathbb{Z}_{p}} \cdots \int_{\mathbb{Z}_{p}} \prod_{i=0}^{n-1}\left(x_{1}+x_{2}+\cdots+x_{k}-\alpha_{i}\right)^{r_{i}} \mathrm{~d} \mu_{0}\left(x_{1}\right) \mathrm{d} \mu_{0}\left(x_{2}\right) \cdots \mathrm{d} \mu_{0}\left(x_{k}\right),
$$

where $n$ is nonnegative integer.

Theorem 1. The numbers $D_{n ; \bar{\alpha}, \bar{r}}^{(k)}$ satisfy the relation

$$
D_{n ; \overline{\boldsymbol{\alpha}}, \overline{\boldsymbol{r}}}^{(k)}=\sum_{i=0}^{|r|} S(n, i ; \overline{\boldsymbol{\alpha}}, \overline{\boldsymbol{r}}) D_{i}^{(k)},
$$


Proof. The generalized Comtet numbers of the first and second kind, $s_{\bar{\alpha}}(n, i ; \overline{\boldsymbol{r}})$ and $S_{\bar{\alpha}}(n, i ; \overline{\boldsymbol{r}})$, (see [14] [15] [16]), are defined, respectively, by

$$
(x ; \bar{\alpha}, \overline{\boldsymbol{r}})_{n}=\sum_{i=0}^{n} s_{\bar{\alpha}}(n, i ; \overline{\boldsymbol{r}}) x^{i},
$$

and

$$
x^{n}=\sum_{i=0}^{n} S_{\bar{\alpha}}(n, i ; \overline{\boldsymbol{r}})(x ; \overline{\boldsymbol{\alpha}}, \overline{\boldsymbol{r}})_{i},
$$

where $(x ; \overline{\boldsymbol{\alpha}}, \overline{\boldsymbol{r}})_{n}=\prod_{i=0}^{n-1}\left(x-\alpha_{i}\right)^{r_{i}}, \overline{\boldsymbol{\alpha}}=\left(\alpha_{0}, \alpha_{1}, \cdots, \alpha_{n-1}\right), \overline{\boldsymbol{r}}=\left(r_{0}, r_{1}, \cdots, r_{n-1}\right)$.

From Equation (10) and using Equation (12), we have

$$
\begin{aligned}
& D_{n ; \overline{\boldsymbol{\alpha}}, \overline{\boldsymbol{r}}}^{(k)}=\int_{\mathbb{Z}_{p}} \int_{\mathbb{Z}_{p}} \cdots \int_{\mathbb{Z}_{p}} \sum_{m=0}^{|r|} s_{\bar{\alpha}}(n, m ; \overline{\boldsymbol{r}})\left(x_{1}+x_{2}+\cdots+x_{k}\right)^{m} \mathrm{~d} \mu_{0}\left(x_{1}\right) \cdots \mathrm{d} \mu_{0}\left(x_{k}\right) \\
& =\sum_{m=0}^{|r|} s_{\bar{\alpha}}(n, m ; \overline{\boldsymbol{r}}) \int_{\mathbb{Z}_{p}} \int_{\mathbb{Z}_{p}} \cdots \int_{\mathbb{Z}_{p}}\left(x_{1}+x_{2}+\cdots+x_{k}\right)^{m} \mathrm{~d} \mu_{0}\left(x_{1}\right) \cdots \mathrm{d} \mu_{0}\left(x_{k}\right) \\
& =\sum_{m=0}^{|r|} s_{\bar{\alpha}}(n, m ; \overline{\boldsymbol{r}}) \int_{\mathbb{Z}_{p}} \int_{\mathbb{Z}_{p}} \cdots \int_{\mathbb{Z}_{p}} \sum_{\ell=0}^{m} S(m, \ell)\left(x_{1}+x_{2}+\cdots+x_{k}\right)_{\ell} \mathrm{d} \mu_{0}\left(x_{1}\right) \cdots \mathrm{d} \mu_{0}\left(x_{k}\right) \\
& =\sum_{m=0}^{|r|} s_{\bar{\alpha}}(n, m ; \overline{\boldsymbol{r}}) \sum_{\ell=0}^{m} S(m, \ell) \int_{\mathbb{Z}_{p}} \int_{\mathbb{Z}_{p}} \cdots \int_{\mathbb{Z}_{p}}\left(x_{1}+x_{2}+\cdots+x_{k}\right)_{\ell} \mathrm{d} \mu_{0}\left(x_{1}\right) \cdots \mathrm{d} \mu_{0}\left(x_{k}\right) .
\end{aligned}
$$

Substituting from Equation (3) into Equation (14) we have

$$
\begin{aligned}
D_{n ; \bar{\alpha}, \bar{r}}^{(k)} & =\sum_{m=0}^{|r|} s_{\bar{\alpha}}(n, m ; \overline{\boldsymbol{r}}) \sum_{\ell=0}^{m} S(m, \ell) D_{\ell}^{(k)} \\
& =\sum_{m=0}^{|r|} \sum_{\ell=0}^{m} s_{\bar{\alpha}}(n, m ; \overline{\boldsymbol{r}}) S(m, \ell) D_{\ell}^{(k)} \\
& =\sum_{\ell=0}^{|r|} \sum_{m=\ell}^{|r|} s_{\bar{\alpha}}(n, m ; \overline{\boldsymbol{r}}) S(m, \ell) D_{\ell}^{(k)} .
\end{aligned}
$$

Since, see [15],

$$
\sum_{m=0}^{|r|} s_{\bar{\alpha}}(n, m ; \overline{\boldsymbol{r}}) S(m, \ell)=S(n, l ; \overline{\boldsymbol{\alpha}}, \overline{\boldsymbol{r}}),
$$

hence we obtain Equation (11).

Next we derive the following theorem which gives a representation of the multiparameter higher order Daehee numbers of the first kind in terms of the generalized multiparameter non central Stirling numbers of the second kind and Stirling number of the first kind, see [1] [10] [17].

Theorem 2. The numbers $D_{n ; \bar{\alpha}, \bar{r}}^{(k)}$ satisfy the relation

$$
D_{n ; \bar{\alpha}, \bar{r}}^{(k)}=\sum_{\ell=0}^{|r|} \frac{S(n, \ell ; \bar{\alpha}, \overline{\boldsymbol{r}}) s(\ell+k, k)}{\left(\begin{array}{c}
\ell+k \\
k
\end{array}\right)} .
$$

Proof. Substituting from Equation (9) into Equation (11) we obtain Equation (17).

\section{Remark 1:}




$$
\begin{aligned}
& \int_{\mathbb{Z}_{p}} \int_{\mathbb{Z}_{p}} \cdots \int_{\mathbb{Z}_{p}} \prod_{i=0}^{n-1}\left(x_{1}+x_{2}+\cdots+x_{k}-\alpha_{i}\right)^{r_{i}} \mathrm{~d} \mu_{0}\left(x_{1}\right) \mathrm{d} \mu_{0}\left(x_{2}\right) \cdots \mathrm{d} \mu_{0}\left(x_{k}\right) \\
& =\sum_{\ell=0}^{|r|} \frac{S(n, \ell ; \overline{\boldsymbol{\alpha}}, \overline{\boldsymbol{r}}) s(\ell+k, k)}{\left(\begin{array}{c}
\ell+k \\
k
\end{array}\right)} .
\end{aligned}
$$

Theorem 3. The numbers $D_{n ; \bar{\alpha}, \bar{r}}^{(k)}$ satisfy the relation

$$
D_{n ; \bar{\alpha}, \bar{r}}^{(k)}=\sum_{\ell=0}^{|r|} s_{\bar{\alpha}}(n, \ell ; \overline{\boldsymbol{r}}) B_{\ell}^{(k)} .
$$

Proof. Using Equation (7) in Equation (11) we have

$$
\begin{aligned}
D_{n ; \overline{\boldsymbol{\alpha}}, \overline{\boldsymbol{r}}}^{(k)} & =\sum_{\ell=0}^{|r|} S(n, \ell ; \overline{\boldsymbol{\alpha}}, \overline{\boldsymbol{r}}) \sum_{i=0}^{\ell} s(\ell, i) B_{i}^{(k)} \\
& =\sum_{\ell=0}^{|r|} \sum_{i=0}^{\ell} S(n, \ell ; \overline{\boldsymbol{\alpha}}, \overline{\boldsymbol{r}}) s(\ell, i) B_{i}^{(k)} \\
& =\sum_{i=0}^{|r|} \sum_{\ell=i}^{|r|} S(n, \ell ; \overline{\boldsymbol{\alpha}}, \overline{\boldsymbol{r}}) s(\ell, i) B_{i}^{(k)} .
\end{aligned}
$$

Substituting from [15, Equation (4.5)] into Equation (20), we obtain Equation (19).

Theorem 4. The numbers $B_{\ell}^{(k)}$ satisfy the relation

$$
B_{n}^{(k)}=\sum_{\ell=0}^{|r|} S_{\bar{\alpha}}(n, \ell ; \overline{\boldsymbol{r}}) D_{\ell ; \bar{\alpha}, \bar{r}}^{(k)} .
$$

Proof. From Equation (19)

$$
D_{n ; \bar{\alpha}, \bar{r}}^{(k)}=\sum_{\ell=0}^{|r|} s_{\bar{\alpha}}(n, \ell ; \overline{\boldsymbol{r}}) B_{\ell}^{(k)},
$$

we can write this equation in the matrix form as follows

$$
\boldsymbol{D}_{\bar{\alpha}, \bar{r}}^{(k)}=\boldsymbol{s}_{\bar{\alpha}}(\overline{\boldsymbol{r}}) \boldsymbol{B}^{(k)},
$$

thus we get

$$
\boldsymbol{S}_{\bar{\alpha}}(\overline{\boldsymbol{r}}) \boldsymbol{D}_{\bar{\alpha}, \bar{r}}^{(k)}=\boldsymbol{S}_{\bar{\alpha}}(\overline{\boldsymbol{r}}) \boldsymbol{s}_{\bar{\alpha}}(\overline{\boldsymbol{r}}) \boldsymbol{B}^{(k)}=\boldsymbol{I} \boldsymbol{B}^{(k)}=\boldsymbol{B}^{(k)},
$$

this matrix form is equivalent to Equation (21).

\section{Multiparameter Higher Order Daehee Polynomials of the First Kind}

The multiparameter higher order Daehee polynomials of the first kind $D_{n ; \bar{\alpha}, \bar{r}}^{(k)}(x)$ are defined by

$$
\begin{aligned}
& D_{n ; \bar{\alpha}, \bar{r}}^{(k)}(x) \\
& =\int_{\mathbb{Z}_{p}} \int_{\mathbb{Z}_{p}} \cdots \int_{\mathbb{Z}_{p}} \prod_{i=0}^{n-1}\left(x_{1}+x_{2}+\cdots+x_{k}+x-\alpha_{i}\right)^{r_{i}} \mathrm{~d} \mu_{0}\left(x_{1}\right) \mathrm{d} \mu_{0}\left(x_{2}\right) \cdots \mathrm{d} \mu_{0}\left(x_{k}\right) .
\end{aligned}
$$

Theorem 5. The polynomials $D_{n ; \bar{\alpha}, \bar{r}}^{(k)}(x)$ satisfy the relation

$$
D_{n ; \overline{\boldsymbol{\alpha}}, \overline{\boldsymbol{r}}}^{(k)}(x)=\sum_{i=0}^{|r|} S(n, i ; \overline{\boldsymbol{\alpha}}, \overline{\boldsymbol{r}}) D_{i}^{(k)}(x) .
$$

Proof. From Equation (24) we have 


$$
\begin{aligned}
& D_{n ; \overline{\boldsymbol{\alpha}}, \overline{\boldsymbol{r}}}^{(k)}(x) \\
& =\int_{\mathbb{Z}_{p}} \int_{\mathbb{Z}_{p}} \cdots \int_{\mathbb{Z}_{p}} \sum_{m=0}^{|r|} s_{\bar{\alpha}}(n, m ; \overline{\boldsymbol{r}})\left(x_{1}+x_{2}+\cdots+x_{k}+x\right)^{m} \mathrm{~d} \mu_{0}\left(x_{1}\right) \cdots \mathrm{d} \mu_{0}\left(x_{k}\right) \\
& =\sum_{m=0}^{|r|} s_{\bar{\alpha}}(n, m ; \overline{\boldsymbol{r}}) \int_{\mathbb{Z}_{p}} \cdots \int_{\mathbb{Z}_{p}}\left(x_{1}+\cdots+x_{k}+x\right)^{m} \mathrm{~d} \mu_{0}\left(x_{1}\right) \cdots \mathrm{d} \mu_{0}\left(x_{k}\right) \\
& =\sum_{m=0}^{|r|} s_{\bar{\alpha}}(n, m ; \overline{\boldsymbol{r}}) \int_{\mathbb{Z}_{p}} \cdots \int_{\mathbb{Z}_{p}} \sum_{\ell=0}^{m} S(m, \ell)\left(x_{1}+\cdots+x_{k}+x\right)_{\ell} \mathrm{d} \mu_{0}\left(x_{1}\right) \cdots \mathrm{d} \mu_{0}\left(x_{k}\right) \\
& =\sum_{m=0}^{|r|} s_{\bar{\alpha}}(n, m ; \overline{\boldsymbol{r}}) \sum_{\ell=0}^{m} S(m, \ell) \int_{\mathbb{Z}_{p}} \cdots \int_{\mathbb{Z}_{p}}\left(x_{1}+\cdots+x_{k}+x\right)_{\ell} \mathrm{d} \mu_{0}\left(x_{1}\right) \cdots \mathrm{d} \mu_{0}\left(x_{k}\right) .
\end{aligned}
$$

Substituting from Equation (5) into Equation (26) we have

$$
\begin{aligned}
D_{n ; \bar{\alpha}, \bar{r}}^{(k)}(x) & =\sum_{m=0}^{|r|} s_{\bar{\alpha}}(n, m ; \bar{r}) \sum_{\ell=0}^{m} S(m, \ell) D_{\ell}^{(k)}(x) \\
& =\sum_{m=0}^{|r|} \sum_{\ell=0}^{m} s_{\bar{\alpha}}(n, m ; \overline{\boldsymbol{r}}) S(m, \ell) D_{\ell}^{(k)}(x) \\
& =\sum_{\ell=0}^{|r|} \sum_{m=\ell}^{|r|} s_{\bar{\alpha}}(n, m ; \overline{\boldsymbol{r}}) S(m, \ell) D_{\ell}^{(k)}(x),
\end{aligned}
$$

substituting from Equation (16) into Equation (27) we obtain Equation (25).

Theorem 6. The polynomials $D_{n ; \bar{\alpha}, \bar{r}}^{(k)}(x)$ satisfy the relation

$$
D_{n ; \bar{\alpha}, \bar{r}}^{(k)}(x)=\sum_{\ell=0}^{|r|} s_{\bar{\alpha}}(n, \ell ; \overline{\boldsymbol{r}}) B_{\ell}^{(k)}(x) .
$$

Proof. Using Equation (7) in Equation (25) we have

$$
\begin{aligned}
D_{n ; \overline{\boldsymbol{\alpha}}, \overline{\boldsymbol{r}}}^{(k)}(x) & =\sum_{\ell=0}^{|r|} S(n, \ell ; \overline{\boldsymbol{\alpha}}, \overline{\boldsymbol{r}}) \sum_{i=0}^{\ell} s(\ell, i) B_{i}^{(k)}(x) \\
& =\sum_{\ell=0}^{|r|} \sum_{i=0}^{\ell} S(n, \ell ; \overline{\boldsymbol{\alpha}}, \overline{\boldsymbol{r}}) s(\ell, i) B_{i}^{(k)}(x) \\
& =\sum_{i=0}^{|r|} \sum_{\ell=i}^{|r|} S(n, \ell ; \overline{\boldsymbol{\alpha}}, \overline{\boldsymbol{r}}) s(\ell, i) B_{i}^{(k)}(x) .
\end{aligned}
$$

Substituting from [15, Equation (4.5)] into Equation (29) we obtain Equation (28).

Theorem 7. The polynomials $B_{\ell}^{(k)}(x)$ satisfy the relation

$$
B_{n}^{(k)}(x)=\sum_{\ell=0}^{|r|} S_{\bar{\alpha}}(n, \ell ; \overline{\boldsymbol{r}}) D_{\ell ; \bar{\alpha}, \bar{r}}^{(k)}(x) .
$$

Proof. From Equation (28)

$$
D_{n ; \bar{\alpha}, \bar{r}}^{(k)}(x)=\sum_{\ell=0}^{|r|} s_{\bar{\alpha}}(n, \ell ; \overline{\boldsymbol{r}}) B_{\ell}^{(k)}(x),
$$

this equation can be written in the following matrix form

$$
\boldsymbol{D}_{\bar{\alpha}, \bar{r}}^{(k)}(x)=\boldsymbol{s}_{\bar{\alpha}}(\overline{\boldsymbol{r}}) \boldsymbol{B}^{(k)}(x) .
$$

We easily have the matrix form

$$
\boldsymbol{S}_{\bar{\alpha}}(\overline{\boldsymbol{r}}) \boldsymbol{D}_{\bar{\alpha}, \bar{r}}^{(k)}(x)=\boldsymbol{S}_{\bar{\alpha}}(\overline{\boldsymbol{r}}) \boldsymbol{s}_{\bar{\alpha}}(\overline{\boldsymbol{r}}) \boldsymbol{B}^{(k)}(x)=\boldsymbol{I} \boldsymbol{B}^{(k)}(x)=\boldsymbol{B}^{(k)}(x) .
$$


This is equivalent to Equation (30).

Moreover some interesting special cases are investigated.

Some special cases:

Case 1: Setting $x_{1}+x_{2}+\cdots+x_{k}=x$ in Equation (10), we obtain

$$
D_{n ; \bar{\alpha}, \bar{r}}=\int_{\mathbb{Z}_{p}}\left(x-\alpha_{0}\right)^{r_{0}}\left(x-\alpha_{1}\right)^{r_{1}} \cdots\left(x-\alpha_{n-1}\right)^{r_{n-1}} \mathrm{~d} \mu_{0}(x)
$$

Corollary 1. The numbers $D_{n ; \bar{\alpha}, \bar{r}}$ satisfy the relation

$$
D_{n ; \bar{\alpha}, \bar{r}}=\sum_{i=0}^{|r|} S(n, i ; \bar{\alpha}, \overline{\boldsymbol{r}}) D_{i} .
$$

Proof. Setting $x_{1}+x_{2}+\cdots+x_{k}=x$ in Equation (11), we obtain Equation (32).

Corollary 2. The numbers $D_{n ; \bar{\alpha}, \bar{r}}$ satisfy the relation

$$
D_{n ; \bar{\alpha}, \bar{r}}=\sum_{\ell=0}^{|r|} s_{\bar{\alpha}}(n, \ell ; \overline{\boldsymbol{r}}) B_{\ell} .
$$

Proof. Setting $x_{1}+x_{2}+\cdots+x_{k}=x$ in Equation (19), we get Equation (33).

Case 2: Setting $r_{i}=1$ in Equation (31) we have

$$
D_{n ; \bar{\alpha}, \bar{r}}=\int_{\mathbb{Z}_{p}}\left(x-\alpha_{0}\right)\left(x-\alpha_{1}\right) \cdots\left(x-\alpha_{n-1}\right) \mathrm{d} \mu_{0}(x) .
$$

Corollary 3. The numbers $D_{n ; \bar{\alpha}}$ satisfy the relation

$$
D_{n ; \bar{\alpha}}=\sum_{i=0}^{n} S(n, i ; \bar{\alpha}) D_{i} .
$$

Proof. Let $r_{i}=1$ in Equation (32), we obtain Equation (35).

Corollary 4. The numbers $D_{n ; \bar{\alpha}}$ satisfy the relation

$$
D_{n ; \bar{\alpha}}=\sum_{\ell=0}^{|r|} s_{\bar{\alpha}}(n, \ell) B_{\ell}
$$

Proof. Setting $r_{i}=1$ in Equation (33), we obtain Equation (36).

Theorem 8.

$$
\int_{\mathbb{Z}_{p}}\left(x-\alpha_{0}\right)\left(x-\alpha_{1}\right) \cdots\left(x-\alpha_{n-1}\right) \mathrm{d} \mu_{0}(x)=\sum_{i=0}^{n}(-1)^{\ell} S(n, i ; \bar{\alpha}) \frac{\ell !}{\ell}, \quad \ell \geq 0 .
$$

Proof. Substituting by $D_{n}=\frac{(-1)^{n} n !}{n+1}$ (see [2] [10]) in Equation (34) and Equation (35), we obtain Equation (37).

Case 3: Setting $r_{i}=1, \alpha_{i}=i$ in Equation (10) we obtain

$$
\begin{aligned}
D_{n ; \overline{\boldsymbol{\alpha}}, \overline{\boldsymbol{r}}}^{(k)} & =\int_{\mathbb{Z}_{p}} \int_{\mathbb{Z}_{p}} \cdots \int_{\mathbb{Z}_{p}} \prod_{i=0}^{n-1}\left(x_{1}+x_{2}+\cdots+x_{k}-i\right) \mathrm{d} \mu_{0}\left(x_{1}\right) \mathrm{d} \mu_{0}\left(x_{2}\right) \cdots \mathrm{d} \mu_{0}\left(x_{k}\right) \\
& =\int_{\mathbb{Z}_{p}} \int_{\mathbb{Z}_{p}} \cdots \int_{\mathbb{Z}_{p}}\left(x_{1}+x_{2}+\cdots+x_{k}\right)_{n} \mathrm{~d} \mu_{0}\left(x_{1}\right) \mathrm{d} \mu_{0}\left(x_{2}\right) \cdots \mathrm{d} \mu_{0}\left(x_{k}\right)=D_{n}^{(k)},
\end{aligned}
$$

and

$$
D_{n}^{(k)}(x)=\sum_{\ell=0}^{n} s(n, \ell) B_{\ell}^{(k)}(x) .
$$




\section{Multiparameter Higher Order Daehee Numbers of the Second Kind}

The multiparameter higher order Daehee numbers of the second kind $\hat{D}_{n ; \bar{\alpha}, \bar{r}}^{(k)}$ are defined by

$$
\hat{D}_{n ; \bar{\alpha}, \bar{r}}^{(k)}=\int_{\mathbb{Z}_{p}} \int_{\mathbb{Z}_{p}} \cdots \int_{\mathbb{Z}_{p}} \prod_{i=0}^{n-1}\left(-x_{1}-x_{2}-\cdots-x_{k}-\alpha_{i}\right)^{r_{i}} \mathrm{~d} \mu_{0}\left(x_{1}\right) \mathrm{d} \mu_{0}\left(x_{2}\right) \cdots \mathrm{d} \mu_{0}\left(x_{k}\right) .(
$$

Theorem 9. The numbers $\hat{D}_{n ; \bar{\alpha}, \bar{r}}^{(k)}$ satisfy the relation

$$
\hat{D}_{n ; \bar{\alpha}, \bar{r}}^{(k)}=\sum_{\ell=0}^{|r|}(-1)^{\ell} S(n, \ell ; \bar{\alpha}, \overline{\boldsymbol{r}}) D_{\ell}^{(k)},
$$

where $(x ; \overline{\boldsymbol{\alpha}}, \overline{\boldsymbol{r}})_{n}=\prod_{i=0}^{n-1}\left(x-\alpha_{i}\right)^{r_{i}}, \overline{\boldsymbol{\alpha}}=\left(\alpha_{0}, \alpha_{1}, \cdots, \alpha_{n-1}\right), \overline{\boldsymbol{r}}=\left(r_{0}, r_{1}, \cdots, r_{n-1}\right)$.

Proof. Using Equation (39) we have

$\hat{D}_{n ; \bar{\alpha}, \bar{r}}^{(k)}$

$=\int_{\mathbb{Z}_{p}} \cdots \int_{\mathbb{Z}_{p}} \sum_{m=0}^{|r|}(-1)^{m} s_{\bar{\alpha}}(n, m ; \overline{\boldsymbol{r}})\left(x_{1}+x_{2}+\cdots+x_{k}\right)^{m} \mathrm{~d} \mu_{0}\left(x_{1}\right) \cdots \mathrm{d} \mu_{0}\left(x_{k}\right)$

$=\sum_{m=0}^{|r|}(-1)^{m} s_{\bar{\alpha}}(n, m ; \overline{\boldsymbol{r}}) \int_{\mathbb{Z}_{p}} \cdots \int_{\mathbb{Z}_{p}}\left(x_{1}+x_{2}+\cdots+x_{k}\right)^{m} \mathrm{~d} \mu_{0}\left(x_{1}\right) \cdots \mathrm{d} \mu_{0}\left(x_{k}\right)$

$=\sum_{m=0}^{|r|}(-1)^{m} S_{\bar{\alpha}}(n, m ; \overline{\boldsymbol{r}}) \int_{\mathbb{Z}_{p}} \cdots \int_{\mathbb{Z}_{p}} \sum_{\ell=0}^{m} S(m, \ell)\left(x_{1}+x_{2}+\cdots+x_{k}\right)_{\ell} \mathrm{d} \mu_{0}\left(x_{1}\right) \cdots \mathrm{d} \mu_{0}\left(x_{k}\right)$

$=\sum_{m=0}^{|r|}(-1)^{m} S_{\bar{\alpha}}(n, m ; \overline{\boldsymbol{r}}) \sum_{\ell=0}^{m} S(m, \ell) \int_{\mathbb{Z}_{p}} \cdots \int_{\mathbb{Z}_{p}}\left(x_{1}+x_{2}+\cdots+x_{k}\right) \mathrm{d} \mu_{0}\left(x_{1}\right) \cdots \mathrm{d} \mu_{0}\left(x_{k}\right)$

$=\sum_{m=0}^{|r|}(-1)^{m} S_{\bar{\alpha}}(n, m ; \bar{r}) \sum_{\ell=0}^{m} S(m, \ell) D_{\ell}^{(k)}$

$=\sum_{m=0}^{|r|} \sum_{\ell=0}^{m}(-1)^{m} s_{\bar{\alpha}}(n, m ; \bar{r}) S(m, \ell) D_{\ell}^{(k)}$

$=\sum_{\ell=0}^{|r|} \sum_{m=\ell}^{|r|}(-1)^{\ell} S_{\bar{\alpha}}(n, m ; \overline{\boldsymbol{r}}) S(m, \ell) D_{\ell}^{(k)}$,

substituting from Equation (16) in Equation (41), then we obtain Equation (40).

Next we derive the following theorem which gives a representation of multiparameter higher order Daehee numbers of the second kind in terms of the generalized multiparameter non-central Stirling numbers of the second kind and Stirling number of the first kind, see [1] [10] [17].

Theorem 10. The numbers $\hat{D}_{n ; \bar{\alpha}, \bar{r}}^{(k)}$ satisfy the relation

$$
\hat{D}_{n ; \overline{\boldsymbol{\alpha}}, \overline{\boldsymbol{r}}}^{(k)}=\sum_{\ell=0}^{|r|}(-1)^{\ell} \frac{S(n, \ell ; \overline{\boldsymbol{\alpha}}, \bar{r}) s(\ell+k, k)}{\left(\begin{array}{c}
\ell+k \\
k
\end{array}\right)} .
$$

Proof. Substituting Equation (9) in Equation (40), we obtain Equation (42).

Remark 2: For $n \in \mathbb{N}$, 


$$
\begin{aligned}
& \int_{\mathbb{Z}_{p}} \cdots \int_{\mathbb{Z}_{p}} \prod_{i=0}^{n-1}\left(-x_{1}-x_{2}-\cdots-x_{k}-\alpha_{i}\right)^{r_{i}} \mathrm{~d} \mu_{0}\left(x_{1}\right) \mathrm{d} \mu_{0}\left(x_{2}\right) \cdots \mathrm{d} \mu_{0}\left(x_{k}\right) \\
& =\sum_{\ell=0}^{|r|}(-1)^{\ell} \frac{S(n, \ell ; \overline{\boldsymbol{\alpha}}, \overline{\boldsymbol{r}}) s(\ell+k, k)}{\left(\begin{array}{c}
\ell+k \\
k
\end{array}\right)} .
\end{aligned}
$$

Theorem 11. The numbers $\hat{D}_{n ; \bar{\alpha}, \bar{r}}^{(k)}$ satisfy the relation

$$
\hat{D}_{n ; \bar{\alpha}, \bar{r}}^{(k)}=\sum_{\ell=0}^{|r|}(-1)^{\ell} s_{\bar{\alpha}}(n, \ell ; \overline{\boldsymbol{r}}) B_{\ell}^{(k)} .
$$

Proof. Substituting Equation (7) in Equation (40) we have

$$
\begin{aligned}
\hat{D}_{n ; \overline{\boldsymbol{\alpha}}, \overline{\boldsymbol{r}}}^{(k)} & =\sum_{\ell=0}^{|r|}(-1)^{\ell} S(n, \ell ; \overline{\boldsymbol{\alpha}}, \overline{\boldsymbol{r}}) \sum_{i=0}^{\ell} s(\ell, i) B_{i}^{(k)} \\
& =\sum_{\ell=0}^{|r|} \sum_{i=0}^{\ell}(-1)^{\ell} S(n, \ell ; \overline{\boldsymbol{\alpha}}, \overline{\boldsymbol{r}}) s(\ell, i) B_{i}^{(k)} \\
& =\sum_{i=0}^{|r|} \sum_{\ell=i}^{|r|}(-1)^{i} S(n, \ell ; \overline{\boldsymbol{\alpha}}, \overline{\boldsymbol{r}}) s(\ell, i) B_{i}^{(k)} .
\end{aligned}
$$

Using [15, Equation (4.5)], we obtain Equation (44).

Theorem 12. The numbers $B_{\ell}^{(k)}$ satisfy the relation

$$
B_{n}^{(k)}=\sum_{\ell=0}^{|r|}(-1)^{\ell} S_{\bar{\alpha}}(n, \ell ; \overline{\boldsymbol{r}}) \hat{D}_{\ell ; \bar{\alpha}, \bar{r}}^{(k)} .
$$

Proof. Equation (44) can be written in a matrix form as

$$
\hat{\boldsymbol{D}}_{\bar{\alpha}, \bar{r}}^{(k)}=\boldsymbol{s}_{\bar{\alpha}}(\overline{\boldsymbol{r}}) \boldsymbol{I}_{1} \boldsymbol{B}^{(k)},
$$

hence we get

$$
\begin{aligned}
& \boldsymbol{S}_{\bar{\alpha}}(\overline{\boldsymbol{r}}) \hat{\boldsymbol{D}}_{\bar{\alpha}, \overline{\boldsymbol{r}}}^{(k)}=\boldsymbol{S}_{\bar{\alpha}}(\overline{\boldsymbol{r}}) \boldsymbol{s}_{\bar{\alpha}}(\overline{\boldsymbol{r}}) \boldsymbol{I}_{1} \boldsymbol{B}^{(k)}=\boldsymbol{I}_{1} \boldsymbol{B}^{(k)}, \\
& \boldsymbol{I}_{1} \boldsymbol{S}_{\bar{\alpha}}(\overline{\boldsymbol{r}}) \hat{\boldsymbol{D}}_{\bar{\alpha}, \overline{\boldsymbol{r}}}^{(k)}=\boldsymbol{I}_{1} \boldsymbol{I}_{1} \boldsymbol{B}^{(k)}=\boldsymbol{I B}^{(k)}=\boldsymbol{B}^{(k)}
\end{aligned}
$$

this is equivalent to Equation (45). Where $\boldsymbol{I}_{1}$ is the diagonal $(n+1) \times(n+1)$ matrix with elements $\left(I_{1}\right)_{i j}=(-1)^{i}, i=j=0,1, \cdots, n$.

\section{Multiparameter Higher Order Daehee Polynomials of the} Second Kind

The multiparameter higher order Daehee polynomials of the second kind $\hat{D}_{n ; \bar{\alpha}, \bar{r}}^{(k)}(x)$ are defined by

$$
\begin{aligned}
& \hat{D}_{n ; \overline{\boldsymbol{\alpha}}, \overline{\boldsymbol{r}}}^{(k)}(x) \\
& =\int_{\mathbb{Z}_{p}} \cdots \int_{\mathbb{Z}_{p}} \prod_{i=0}^{n-1}\left(-x_{1}-x_{2}-\cdots-x_{k}+x-\alpha_{i}\right)^{r_{i}} \mathrm{~d} \mu_{0}\left(x_{1}\right) \mathrm{d} \mu_{0}\left(x_{2}\right) \cdots \mathrm{d} \mu_{0}\left(x_{k}\right) .
\end{aligned}
$$

Theorem 13. The polynomials $\hat{D}_{n ; \bar{\alpha}, \bar{r}}^{(k)}(x)$ satisfy the relation

$$
\hat{D}_{n ; \bar{\alpha}, \bar{r}}^{(k)}(x)=\sum_{i=0}^{|r|}(-1)^{i} S(n, i ; \bar{\alpha}, \overline{\boldsymbol{r}}) D_{i}^{(k)}(-x) .
$$


Proof. Using Equation (48) we have

$$
\begin{aligned}
\hat{D}_{n ; \bar{\alpha}, \bar{r}}^{(k)}(x) & =\int_{\mathbb{Z}_{p}} \cdots \int_{\mathbb{Z}_{p}} \sum_{m=0}^{|r|}(-1)^{m} S_{\bar{\alpha}}(n, m ; \overline{\boldsymbol{r}})\left(x_{1}+x_{2}+\cdots+x_{k}-x\right)^{m} \mathrm{~d} \mu_{0}\left(x_{1}\right) \cdots \mathrm{d} \mu_{0}\left(x_{k}\right) \\
& =\sum_{m=0}^{|r|}(-1)^{\ell} S_{\bar{\alpha}}(n, m ; \bar{r}) \int_{\mathbb{Z}_{p}} \cdots \int_{\mathbb{Z}_{p}}\left(x_{1}+x_{2}+\cdots+x_{k}-x\right)^{m} \mathrm{~d} \mu_{0}\left(x_{1}\right) \cdots \mathrm{d} \mu_{0}\left(x_{k}\right) \\
& =\sum_{m=0}^{|r|}(-1)^{\ell} S_{\bar{\alpha}}(n, m ; \bar{r}) \int_{\mathbb{Z}_{p}} \cdots \int_{\mathbb{Z}_{p}} \sum_{\ell=0}^{m} S(m, \ell)\left(x_{1}+x_{2}+\cdots+x_{k}-x\right)_{\ell} \mathrm{d} \mu_{0}\left(x_{1}\right) \cdots \mathrm{d} \mu_{0}\left(x_{k}\right) \\
& =\sum_{m=0}^{|r|}(-1)^{\ell} S_{\bar{\alpha}}(n, m ; \bar{r}) \sum_{\ell=0}^{m} S(m, \ell) \int_{\mathbb{Z}_{p}} \cdots \int_{\mathbb{Z}_{p}}\left(x_{1}+x_{2}+\cdots+x_{k}-x\right)_{\ell} \mathrm{d} \mu_{0}\left(x_{1}\right) \cdots \mathrm{d} \mu_{0}\left(x_{k}\right) \\
& =\sum_{m=0}^{|r|}(-1)^{\ell} S_{\bar{\alpha}}(n, m ; \bar{r}) \sum_{\ell=0}^{m} S(m, \ell) D_{\ell}^{(k)}(-x) \\
& =\sum_{m=0}^{|r|} \sum_{\ell=0}^{m}(-1)^{\ell} S_{\bar{\alpha}}(n, m ; \overline{\boldsymbol{r}}) S(m, \ell) D_{\ell}^{(k)}(-x) \\
& =\sum_{\ell=0}^{|r|} \sum_{m=\ell}^{r \mid}(-1)^{\ell} s_{\bar{\alpha}}(n, m ; \bar{r}) S(m, \ell) D_{\ell}^{(k)}(x) .
\end{aligned}
$$

Substituting from Equation (16) into Equation (50), we obtain Equation (49).

Theorem 14. The polynomials $\hat{D}_{n ; \bar{\alpha}, \bar{r}}^{(k)}(x)$ satisfy the relation

$$
\hat{D}_{n ; \bar{\alpha}, \bar{r}}^{(k)}(x)=\sum_{\ell=0}^{|r|}(-1)^{\ell} s_{\bar{\alpha}}(n, \ell ; \overline{\boldsymbol{r}}) B_{\ell}^{(k)}(-x) .
$$

Proof. Using Equation (7) in Equation (49), we have

$$
\begin{aligned}
\hat{D}_{n ; \overline{\boldsymbol{\alpha}}, \overline{\boldsymbol{r}}}^{(k)}(x) & =\sum_{\ell=0}^{|r|}(-1)^{\ell} S(n, \ell ; \overline{\boldsymbol{\alpha}}, \overline{\boldsymbol{r}}) \sum_{i=0}^{\ell} s(\ell, i) B_{i}^{(k)}(-x) \\
& =\sum_{\ell=0}^{|r|} \sum_{i=0}^{\ell}(-1)^{\ell} S(n, \ell ; \overline{\boldsymbol{\alpha}}, \overline{\boldsymbol{r}}) s(\ell, i) B_{i}^{(k)}(-x) \\
& =\sum_{i=0}^{|r|} \sum_{\ell=i}^{|r|}(-1)^{i} S(n, \ell ; \overline{\boldsymbol{\alpha}}, \overline{\boldsymbol{r}}) s(\ell, i) B_{i}^{(k)}(-x) .
\end{aligned}
$$

Substituting from [15, Equation (4.5)] into Equation (52), we obtain Equation (51).

Next we derive some important special cases.

\section{Some special cases:}

Case 1: Setting $-x_{1}-x_{2}-\cdots-x_{k}=-x$ in Equation (39), we obtain

$$
\hat{D}_{n ; \overline{\boldsymbol{\alpha}}, \overline{\boldsymbol{r}}}=\int_{\mathbb{Z}_{p}}\left(-x-\alpha_{0}\right)^{r_{0}}\left(-x-\alpha_{1}\right)^{r_{1}} \cdots\left(-x-\alpha_{n-1}\right)^{r_{n-1}} \mathrm{~d} \mu_{0}(x) .
$$

Corollary 5. The numbers $\hat{D}_{n ; \bar{\alpha}, \bar{r}}$ satisfy the relation

$$
\hat{D}_{n ; \bar{\alpha}, \overline{\boldsymbol{r}}}=\sum_{i=0}^{|r|}(-1)^{i} S(n, i ; \overline{\boldsymbol{\alpha}}, \overline{\boldsymbol{r}}) D_{i} .
$$

Corollary 6. The numbers $\hat{D}_{n ; \bar{\alpha}, \bar{r}}$ satisfy the relation

$$
\hat{D}_{n ; \bar{\alpha}, \bar{r}}=\sum_{\ell=0}^{|r|}(-1)^{\ell} s_{\bar{\alpha}}(n, \ell ; \bar{r}) B_{\ell} .
$$

Case 2: Setting $r_{i}=1$ in Equation (53), we obtain

$$
\hat{D}_{n ; \bar{\alpha}}=\int_{\mathbb{Z}_{p}}\left(-x-\alpha_{0}\right)\left(-x-\alpha_{1}\right) \cdots\left(-x-\alpha_{n-1}\right) \mathrm{d} \mu_{0}(x) .
$$


Corollary 7. The numbers $\hat{D}_{n ; \bar{\alpha}}$ satisfy the relation

$$
\hat{D}_{n ; \bar{\alpha}}=\sum_{i=0}^{n}(-1)^{i} S(n, i ; \bar{\alpha}) D_{i} .
$$

Corollary 8. The numbers $\hat{D}_{n ; \bar{\alpha}}$ satisfy the relation

$$
\hat{D}_{n ; \bar{\alpha}}=\sum_{\ell=0}^{|r|}(-1)^{\ell} s_{\bar{\alpha}}(n, \ell) B_{\ell}
$$

Theorem 15.

$$
\begin{aligned}
& \int_{\mathbb{Z}_{p}}\left(-x-\alpha_{0}\right)\left(-x-\alpha_{1}\right) \cdots\left(-x-\alpha_{n-1}\right) \mathrm{d} \mu_{0}(x) \\
& =\sum_{i=0}^{n}(-1)^{\ell} S(n, i ; \overline{\boldsymbol{\alpha}}) \frac{\ell !}{\ell+1}, \quad \ell \geq 0 .
\end{aligned}
$$

Proof. Substituting by $\hat{D}_{n}=\frac{(-1)^{n} n !}{n+1}$ (see [18]) in Equation (56) and Equation (57), we obtain Equation (58).

\section{Conclusion}

In this paper we define the multiparameter higher order Daehee numbers and polynomials of the first and second kind. Some new results for these numbers and polynomials are derived. Furthermore, some interesting special cases of the multiparameter higher order Daehee and Bernoulli numbers and polynomials are deduced.

\section{Acknowledgements}

We thank the Editor and the referee for their comments.

\section{References}

[1] Kim, D.S., Kim, T., Lee, S.H. and Seo, J.-J. (2014) Higher-Order Daehee Numbers and Polynomials. International Journal of Mathematical Analysis, 8, 273-283. https://doi.org/10.12988/ijma.2014.4118

[2] Kim, D.S., Kim, T., Lee, S.H. and Seo, J.-J. (2013) A Note on the lambda Daehee Polynomials. International Journal of Mathematical Analysis, 7, 3069-3080. https://doi.org/10.12988/ijma.2013.311264

[3] Kim, D.S., Kim, T., Lee, S.H. and Seo, J.-J. (2013) A Note on Twisted $\lambda$ Daehee Polynomials. Applied Mathematical Sciences, 7, 7005-7014. https://doi.org/10.12988/ams.2013.311635

[4] Kim, D.S. and Kim, T. (2013) Daehee Numbers and Polynomials. Applied Mathematical Sciences, 7, 5969-5976. https://doi.org/10.12988/ams.2013.39535

[5] Kim, T. and Simsek, Y. (2008) Analytic Continuation of the Multiple Daehee q-1Functions Associated with Daehee Numbers. Russian Journal of Mathematical Physics, 15, 58-65. https://doi.org/10.1134/S106192080801007X

[6] Kimura, N. (2003) On Universal Higher Order Bernoulli Numbers and Polynomials. Report of the Research Institute of Industrial Technology, Nihon University, No. 70 .

[7] Liu, G.-D. and Srivastava, H.M. (2006) Explicit Formulas for the Nörlund Polynomials $\mathrm{B}(\mathrm{x}) \mathrm{n}$ and $\mathrm{b}(\mathrm{x}) \mathrm{n}$. Computers and Mathematics with Applications, 51, 1377 1384. 
[8] Ozden, H., Cangul, N. and Simsek, Y. (2009) Remarks on q-Bernoulli Numbers Associated with Daehee Numbers. Advanced Studies in Contemporary Mathematics, 18, 41-48.

[9] Wang, W. (2010) Generalized Higher Order Bernoulli Number Pairs and Generalized Stirling Number Pairs. Journal of Mathematical Analysis and Applications, 364, 255-274.

[10] El-Desouky, B.S. and Mustafa, A. (2016) New Results on Higher-Order Daehee and Bernoulli Numbers and Polynomials. Advances in Difference Equations, 2016, 32. https://doi.org/10.1186/s13662-016-0764-Z

[11] Carlitz, L. (1961) A Note on Bernoulli and Euler Polynomials of the Second Kind. Scripta Mathematica, 25, 323-330.

[12] Comtet, L. (1974) Advanced Combinatorics. Reidel, Dordrecht. https://doi.org/10.1007/978-94-010-2196-8

[13] Dolgy, D.V., Kim, T., Lee, B. and Lee, S.H. (2013) Some New Identities on the Twisted Bernoulli and Euler Polynomials. Journal of Computational Analysis and Applications, 14, 441-451.

[14] El-Desouky, B.S. (1994) The Multiparameter Non-Central Stirling Numbers. Fibonacci Quarterly, 32, 218-225.

[15] El-Desouky, B.S. and Cakić, N.P. (2011) Generalized Higher Order Stirling Numbers. Mathematical and Computer Modelling, 54, 2848-2857. https://doi.org/10.1016/j.mcm.2011.07.005

[16] El-Desouky, B.S., Cakić, N.P. and Mansour, T. (2010) Modified Approach to Generalized Stirling Numbers via Differential Operators. Applied Mathematics Letters, 23, 115-120.

[17] Gould, H.W. (1972) Explicit Formulas for Bernoulli Numbers. American Mathematical Monthly, 79, 44-51. https://doi.org/10.2307/2978125

[18] El-Desouky, B.S. and Mustafa, A. (2015) New Results and Matrix Representation for Daehee and Bernoulli Numbers and Polynomials. Applied Mathematical Sciences, 9, 3593-3610. https://doi.org/10.12988/ams.2015.53282

Submit or recommend next manuscript to SCIRP and we will provide best service for you:

Accepting pre-submission inquiries through Email, Facebook, LinkedIn, Twitter, etc. A wide selection of journals (inclusive of 9 subjects, more than 200 journals)

Providing 24-hour high-quality service

User-friendly online submission system

Fair and swift peer-review system

Efficient typesetting and proofreading procedure

Display of the result of downloads and visits, as well as the number of cited articles Maximum dissemination of your research work

Submit your manuscript at: http://papersubmission.scirp.org/

Or contact am@scirp.org 